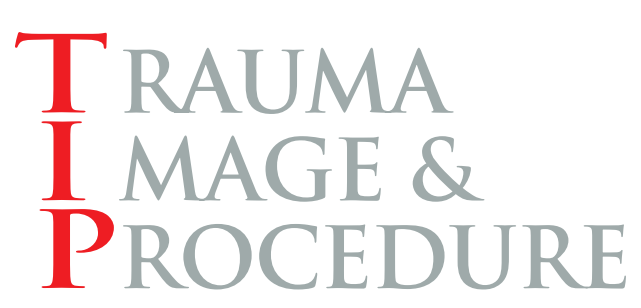

How to Do It in Trauma

Trauma Image Proced 2021;6(1):32-35

https://doi.org/10.24184/tip.2021.6.1.32

elSSN 2508-8033 • pISSN 2508-5298

\title{
Unusual complications of the central venous catheterization in trauma patients
}

Received October 21, 2021

Revised October 22, 2021

Accepted October 23, 2021

\author{
Correspondence to \\ Dong Hun Kim \\ Department of Trauma Surgery, Trauma \\ Center, Dankook University Hospital, \\ 201 Manghyang-ro, Dongnam-gu, \\ Cheonan 31116, Korea \\ Tel: +82-41-550-3042 \\ Fax: +82-41-550-0039 \\ E-mail:saint7331@gmail.com
}

\section{Yoonjung Heo, Dong Hun Kim \\ Department of Trauma Surgery, Trauma Center, Dankook University Hospital, Cheonan, Korea}

Central venous catheters (CVCs) are commonly used in patients with trauma. The placement and removal of the CVCs can result in various complications regardless of the skill of the professionals. Herein, two cases of rare complications are presented- an inadvertent subclavian artery catheterization and a cerebral air embolism after a CVC removal. Moreover, practical solutions for each complication are provided in detail.

Key Words: Central venous catheters, Subclavian artery, Vascular closure devices, Air embolism, Hyperbaric oxygenation

\section{Introduction}

Central venous catheters (CVCs) are used to administer massive fluid, blood products, and multiple parenteral medications (e.g., vasoconstrictors or sedatives) in the emergent setting. CVC placement requires proper training and is not without adverse events even when performed by skilled operators. The mechanical complications of CVCs mostly arise during punctures. However, delayed complications associated with maintenance or removal of the catheter also occur (1).

\section{Case presentation}

\section{Case 1}

A 68-year-old female patient presented with multiple deep lacerations in both of the wrists, abdomen, and scalp from suicidal attempts. Upon arrival, the patient was un-

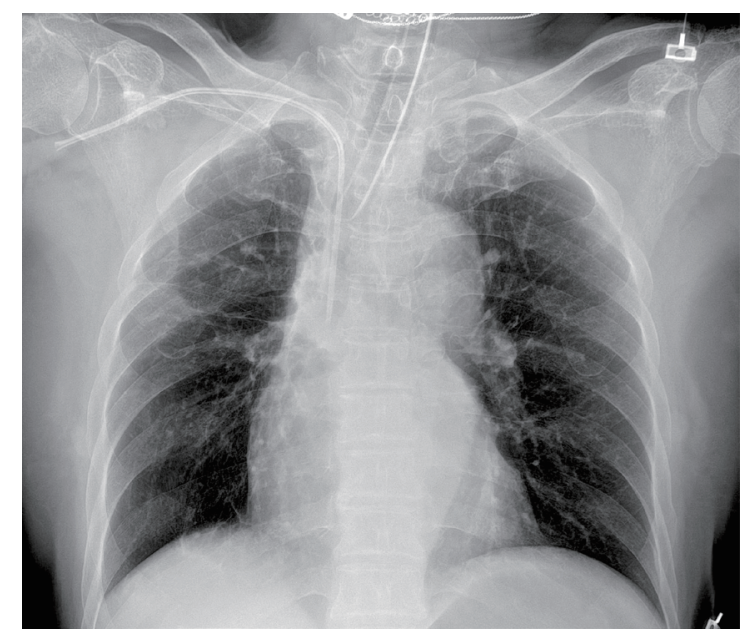

Fig. 1. A portable chest radiograph, which is showing the tip location of the central venous catheter. The ideal location for the tip is in the superior right cavoatrial junction. The radiograph is deceiving as if the catheter is in the right position because of the low blood pressure of the patient.

(C)This is an Open Access article distributed under the terms of the Creative Commons Attribution Non-Commercial License (http://creativecommons.org/licenses/by-nc/4.0) which permits unrestricted noncommercial use, distribution, and reproduction in any medium, provided the original work is properly cited.

Copyright (c) 2021 Korean Association for Research, Procedures and Education on Trauma. All rights reserved. 

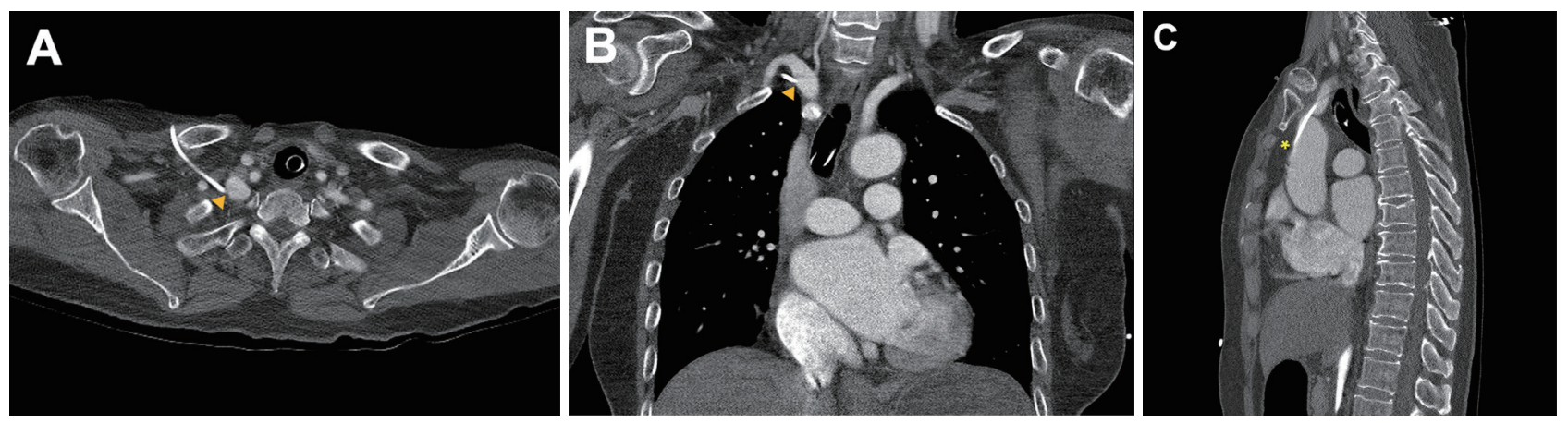

Fig. 2. Preprocedure images of the chest computed tomography showing inadvertent subclavian artery catheterization: axial (A), coronal (B), and sagittal (C) views. The arteriotomy site (arrowhead) is between the ostia of the right internal mammary and vertebral arteries with the tip (asterisk) of the catheter lying in the ascending aorta.
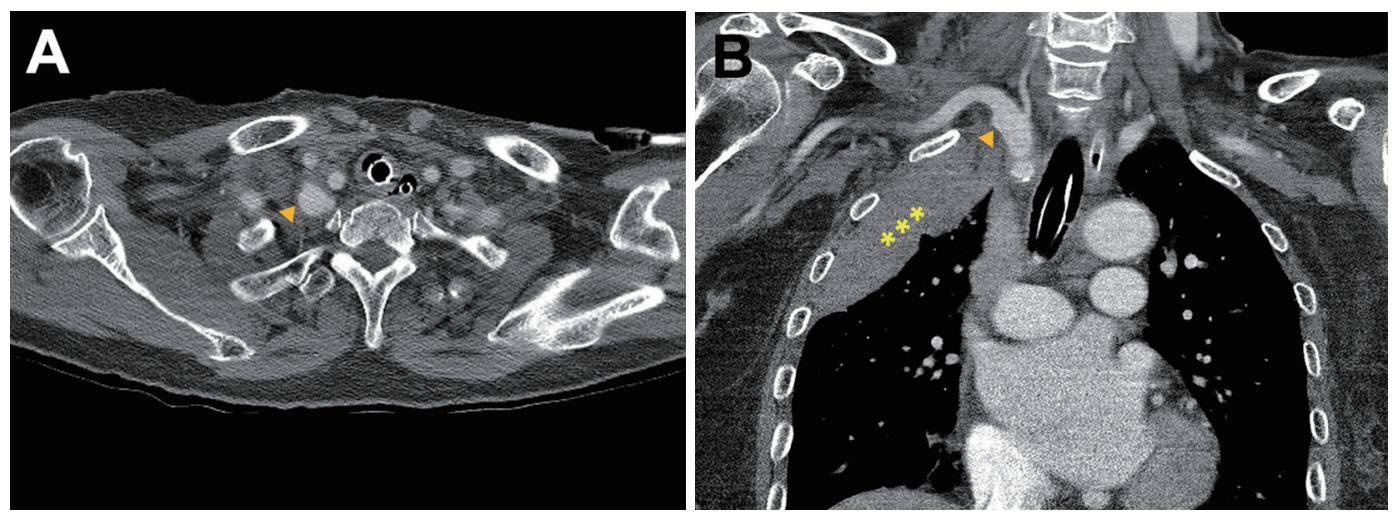

Fig. 3. Postprocedure images (1 h later) of the chest computed tomography showing the patient's state of the right subclavian artery-axial (A) and coronal (B) views. No extravasation, thrombus, or dissection flaps are seen nearby the previous arteriotomy site (arrowhead), which confirms procedural success. An extrapleural hematoma (asterisks) of $100 \times 30 \mathrm{~mm}$ is observed in the right upper part of the extrapleural space, which was created while exchanging the catheter to a vascular closure device for a few seconds. The hematoma did not increase and dissolved afterward.

stable with a blood pressure of 70/30 $\mathrm{mmHg}$ and a heart rate of 133 beats per min with a Glasgow Coma Scale (GCS) score of 6. A 7-Fr CVC was inserted via the right subclavian approach for a resuscitation purpose. A postprocedural chest radiograph showed proper catheter tip placement (Fig. 1). However, unintended CVC placement in the subclavian artery (SCA) was identified later using chest computed tomography (CT) scans (Fig. 2). The distance from the skin surface to the arteriotomy was 3.8 $\mathrm{cm}$. A decision was made to use a vascular closure device (VCD; Femoseal ${ }^{\mathrm{TM}}$, Terumo, NJ, USA) because the SCA could not be manually compressed. The SCA was successfully closed by endovascular VCD deployment (Video 1). A follow-up CT demonstrated the intact SCA without bleeding (Fig. 3).

\section{Case 2}

A 16-year-old male patient with a grade II traumatic spleen laceration was admitted to the intensive care unit. A 7-Fr CVC was placed in the right subclavian vein to aid in resuscitation which was removed 4 days later. The removal was performed while the patient was sitting. After a few seconds, the patient showed blurred vision, seizure, and altered mental status with a decreased GCS of 11. A brain CT was immediately performed and a cerebral air embolism (CAE) was strongly suspected (Fig. 4A). The patient was placed in the Trendelenburg position to prevent further air travel and underwent hyperbaric oxygen therapy (HBOT), which provided $100 \%$ oxygen at $2 \mathrm{~kg} / \mathrm{cm}^{2}$. The patient made a complete neurological recovery after three cycles (50, 50, and $40 \mathrm{~min}$; total=140 $\mathrm{min}$ ) of HBOT (Fig. $4 \mathrm{~B})$. 

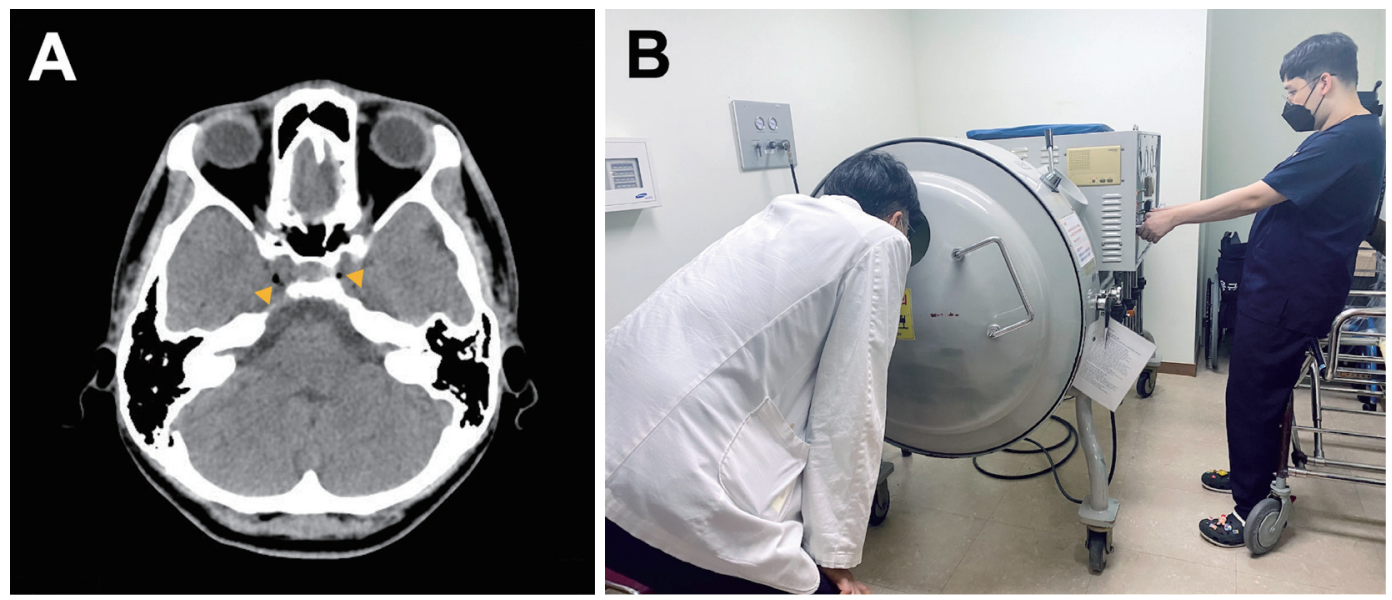

Fig. 4. Noncontrast brain computed tomography (A) shows gas bubbles within the cavernous sinuses (arrowheads), which suggests cerebral air embolism. The patient underwent hyperbaric oxygen therapy in a monoplace chamber (B) while a trauma team monitored and provided care.

\section{Discussion}

An accidental arterial puncture occurs in $2.7 \%$ of the subclavian approaches (2); it can be unrecognized in patients with hypotension with reduced pulsatile flow. Prompt recognition and intervention are essential as prolonged arterial cannulation can result in bleeding, thrombosis, stroke, limb ischemia, neurologic deficit, or even mortality. Treatment options include open surgical repair and endovascular procedures (e.g., embolization, stent insertion, balloon inflation, or use of VCDs) $(3,4)$.

The VCD seals the site of the arteriotomy mechanically by sandwiching two bioabsorbable discs (3). Although the off-label use of VCDs to repair vessels other than a femoral artery lacks data, their use is still increasing (24). According to a meta-analysis, the overall repair success rate of the iatrogenic thoracocervical arterial injuries via VCDs was $95.7 \%$ (4). The current case demonstrates that the VCD is a safe and effective option for accidental CVC cannulation in the SCA. However, the treatment strategies should be tailored on a case-by-case basis, which considers the patient's hemodynamic status, puncture site, underlying arterial conditions, and a caliber of the CVCs.

The incidence of an air embolism while insertion, manipulation, and removal of the CVCs is $0.03 \%-2 \%$ (5). The CAE is a preventable complication if precautionary measures are followed during CVC removal-the head-down position, the Valsalva maneuver, and an airtight dressing (6). Relevant imaging is needed when acute neurological

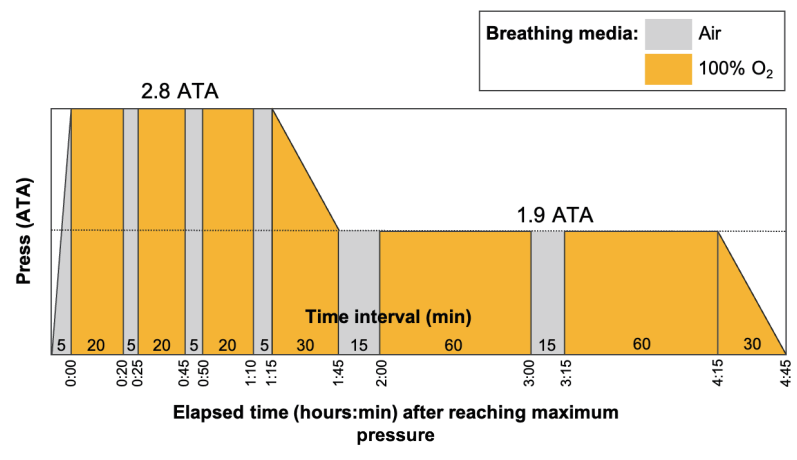

Fig. 5. The United States Navy Table 6 consists of four oxygen cycles of 2.8 atmospheres absolute (ATA) under $100 \%$ oxygen, which can last 20-30 min and two oxygen cycles of 1.9 ATA, which can last $60 \mathrm{~min}$. The total elapsed time is $285 \mathrm{~min}$.

symptoms occur after such procedures for early diagnosis and treatment. The HBOT, according to the United States Navy Table 6 (Fig. 5), is currently considered the standard regimen for CAE treatment $(5,6)$. While mortality of CAE reaches up to $20 \%, 75 \%$ of the survivors are reported to have mild to no disabilities with prompt HBOT treatment (6).

This report is believed to offer important lessons to all professionals who come across CVCs in daily clinical scenes. Knowing how to prevent, recognize, and manage rare complications concerned with the CVCs would decrease the chances of causing harm to patients who are already in their extremis due to polytraumas. 


\section{Conflict of interest}

No potential conflict of interest relevant to this article was reported.

\section{Supplementary material}

Supplementary data including one video can be found with this article online at www.traumaimpro.org.

Video 1. Endovascular deployment of Femoseal ${ }^{\mathrm{TM}}$ to the right subclavian artery. A 0.038-in Femoseal ${ }^{\mathrm{TM}}$ guidewire was advanced through the inadvertently placed subclavian artery catheter. The catheter was then removed and an 8-Fr vascular closure device was deployed over the guidewire. Correct intra-arterial positioning of the device was confirmed by blood flow visualization into the transparent tube. The total procedure time was approximately $2 \mathrm{~min}$.

\section{References}

1. Roldan CJ, Paniagua L. Central venous catheter intravascular malpositioning: causes, prevention, diag- nosis, and correction. West J Emerg Med. 2015;16(5): 658-64.

2. Cohen JE, Moshe Gomori J, Anner H, Itshayek E. Inadvertent subclavian artery cannulation treated by percutaneous closure. J Clin Neurosci. 2014;21(11): 1973-5.

3. Mousa AY, Abu-Halimah S, Nanjundappa A, AbuRahma AF. Inadvertent subclavian artery cannulation and options for management. Vascular. 2015;23(2):132-7.

4. Makris GC, Patel R, Little M, Tyrrell C, Sutcliffe J, Allouni K, et al. Closure devices for iatrogenic thoraco-cervical vascular injuries. Cardiovasc Intervent Radiol. 2017;40(3):381-7.

5. Murphy RP, Donnellan J. A high-pressure solution for a high-pressure situation: management of cerebral air embolism with hyperbaric oxygen therapy. Cureus. 2019;11(9):e5559.

6. Chuang DY, Sundararajan S, Sundararajan VA, Feldman DI, Xiong W. Accidental air embolism. Stroke. 2019;50(7):e183-6. 